\title{
Uma Plataforma para Redução e Separação de Ruído em Drones Equipados com Sensores Sonoros
}

\author{
Luís Augusto Silva ${ }^{1}$, Francisco García Encinas ${ }^{1}$, Bruno Alves da Silva ${ }^{3}$, \\ André Sales Mendes ${ }^{1}$, Valderi R. Q. Leithardt ${ }^{2}$, Gabriel Villarrubia González ${ }^{1}$ \\ ${ }^{1}$ Expert Systems and Applications Lab - ESALAB \\ Facultad de Ciencias, Universidad de Salamanca \\ Plaza de los Caídos s/n, 37008 Salamanca, España \\ ${ }^{2}$ VALORIZA, Research Center for Endogenous Resources Valorization \\ Instituto Politécnico de Portalegre, 7300-555 Portalegre, Portugal \\ ${ }^{3}$ Laboratório de Sistemas Embarcados e Distribuídos \\ Universidade do Vale do Itajaí (UNIVALI), Itajaí, Brasil, 88302-901 \\ \{luisaugustos, frangaren, andremendes, gvg\}@usal.es \\ silvabruno@edu.univali.br, valderi@ipportalegre.pt
}

\begin{abstract}
Resumo. A utilização de drones está aumentando para os novos campos de aplicações, desde a agricultura à segurança. Uma destas aplicações é a medição sonora ou audiovisual em áreas de difícil acesso. Em este trabalho apresentamos a aplicação e o algoritmo que emprega mecanismos de Blind Source Separation (BSS) para a separação das fontes de ruído. $O$ algoritmo utiliza a relação entre a emissão sonora e as fontes de ruído em função das variáveis, tais como potência do motor, rotações por minuto, ou tipo de hélice.
\end{abstract}

\section{Introdução}

Atualmente, os drones são utilizados em uma infinidade de aplicações civis e militares, sendo uma realidade inquestionável. Estes tipos de veículos estão se tornando mais baratos, mais precisos e mais fáceis de utilizar por qualquer usuário. Principalmente, dentro das aplicações civis, tornou-se uma ferramenta tanto para o lazer quanto para o trabalho. Muitas vezes, empresas especializadas em controle de ruído se deparam com áreas de difícil acesso para medir os níveis sonoros produzidos por indústrias ou máquinas de alto porte. A proposta deste trabalho é utilizar drones para abordar fontes de ruído de difícil acesso e utilizar algoritmos para separar o som do drone da fonte gravada. Um dos objetivos é transmitir o sinal do equipamento de gravação de áudio para o operador do drone. Para resolver o problema de eliminar ou separar o ruído de drone, existem técnicas como Blind Source Separation (BSS) [Wang and Cavallaro 2020] que, por sua vez, faz uso do algoritmo de Singular Spectrum Analysis (SSA).

\section{Método Proposto}

Durante as últimas décadas, muita atenção tem sido dada à separação das fontes de ruido, em particular aos casos cegos, os quais as fontes e o processo de união são desconhecidos, enquanto apenas as gravações de todo o conjunto estão disponíveis. Em várias situações, é desejável recuperar todas as fontes gravadas, ou pelo menos segregar uma determinada 
fonte. Além disso, é útil identificar todo entorno da gravação para revelar as informações presentes na gravação física [Ma et al. 2010, Golyandina and Zhigljavsky 2013].

A arquitetura proposta visa abordar os vários problemas que têm tais sistemas, como problemas de comunicação, planejamento de rotas, sincronização e processamento de sinais. A principal característica desta arquitetura é a capacidade de lidar com e incluir novas características e adaptar-se no futuro a novos ambientes e novos ambientes como em [Leithardt et al. 2020]. A arquitetura proposta para este trabalho de pesquisa é apresentada na Figura 1.

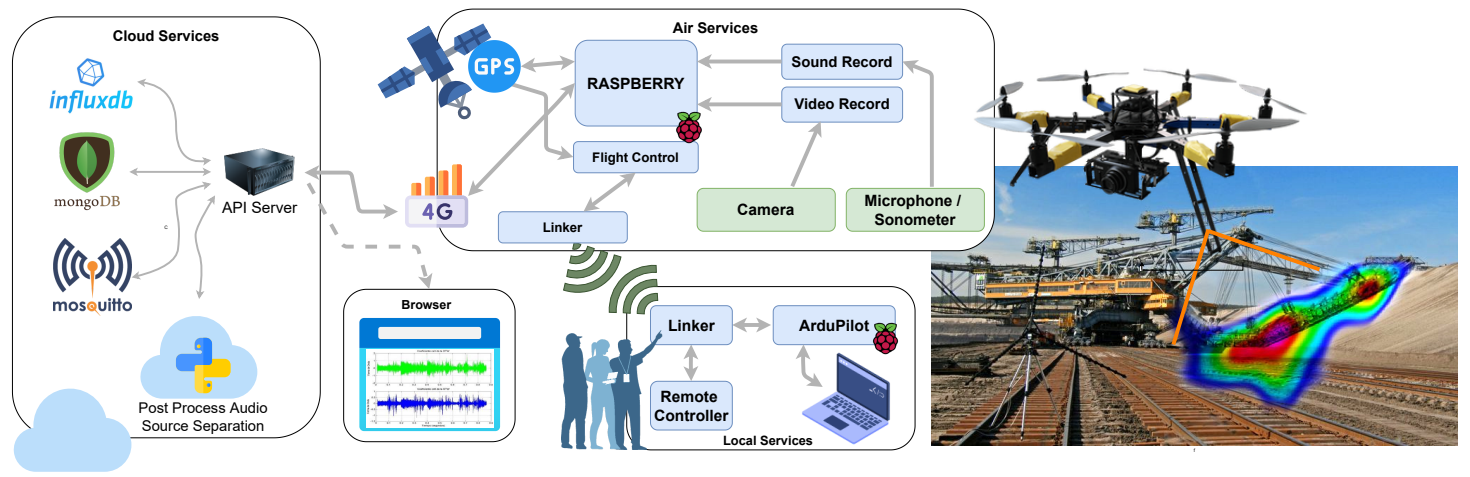

Figure 1. Arquitetura Proposta.

O sistema proposto separa o ruido do drone de uma fonte desconhecida utilizando apenas um microfone. O conhecimento sobre o ruido emitido do drone pode ser explorado para a separação dos dois. Para resolvê-lo, propõe-se usar a análise do espectro singular para separar a observação em seus principais componentes e por fim fazer o processo de reconstrução de ambas as fontes. Além disso, um dos desafios é o processamento e analise dos sinais de áudio em tempo real, o qual deve ser abordado com o uso de paralelismo, utilizando a tecnologia Compute Unified Device Architecture (CUDA) que utiliza o processamento paralelo rápido em unidades de processamento gráfico (GPUs).

\section{Considerações Finais}

Um drone comercial DJI Mavic Air foi utilizado para provas iniciais e com isso foi possível analisar preliminarmente o ruído produzido pelo drone, sendo o ruído previsível e dependente da velocidade angular dos motores. Para continuidade deste trabalho deve-se provar diferentes algoritmos que explorem as características do ruído produzido.

\section{References}

Golyandina, N. and Zhigljavsky, A. (2013). Singular Spectrum Analysis for time series. Springer Science \& Business Media.

Leithardt, V., Santos, D., Silva, L., Viel, F., Zeferino, C., and Silva, J. (2020). A solution for dynamic management of user profiles in iot environments. IEEE Latin America Transactions, 18(07):1193-1199.

Ma, H.-G., Jiang, Q.-B., Liu, Z.-Q., Liu, G., and Ma, Z.-Y. (2010). A novel blind source separation method for single-channel signal. Signal Processing, 90(12):3232 - 3241.

Wang, L. and Cavallaro, A. (2020). A blind source separation framework for ego-noise reduction on multi-rotor drones. IEEE/ACM Transactions on Audio, Speech, and Language Processing, 28:2523-2537. 